\title{
JOGOS DIGITAIS APLICADOS À PROMOÇÃO DO AUTOCUIDADO EM SAÚDE NO ESCOLAR: UMA REVISÃO INTEGRATIVA
}

\author{
Maína Hemann Strack, Universidade Federal de Ciências da Saúde de Porto Alegre- \\ mainahs@ufcspa.edu.br
}

Mariana da Silva Bauer, Universidade Federal de Ciências da Saúde de Porto Alegre - marianasb@ufcspa.edu.br

Luciana Bisio Mattos, Universidade Federal de Ciências da Saúde de Porto Alegrelucianabisiomattos@gmail.com

Silvio Cesar Cazella, Universidade Federal de Ciências da Saúde de Porto Alegresilvioc@ufcspa.edu.br

Cleidilene Ramos Magalhães, Universidade Federal de Ciências da Saúde de Porto Alegre-cleidirm@ufcspa.edu.br

Resumo. Frente à crescente utilização dos serious games como ferramenta educacional, o presente artigo apresenta uma revisão integrativa que teve como objetivo: mapear a utilização de jogos digitais entre escolares na promoção do autocuidado em saúde. O foco do mapeamento foi na análise da contribuição dos jogos no aprendizado, aquisição de conhecimento, desenvolvimento de competências e na própria reflexão sobre autocuidado. A revisão foi realizada a partir de 7 bases eletrônicas. Incluiu-se os estudos publicados nos últimos 5 anos, originais e que tivessem como foco a utilização de jogos digitais com escolares, nas temáticas autocuidado e promoção de saúde. Dos 685 estudos revisados, 05 foram incluídos. A despeito do grande volume de publicações utilizando-se de serious games, verificouse um número reduzido relacionando jogos digitais com escolares, autocuidado e promoção de saúde. Todavia, há indicativos do grande potencial dos jogos digitais no ambiente escolar para promoção de saúde, principalmente em relação ao desenvolvimento de competências para o autocuidado.

Palavras-chaves: jogos de vídeo; saúde escolar; promoção da saúde; autocuidado; competências.

\section{SERIOUS GAMES FOR SELF-CARE PROMOTION IN HEALTH AT THE SCHOOL ENVIRONMENT: AN INTEGRATIVE REVIEW}

\begin{abstract}
Faced with the increasing use of serious games as an educational tool, this paper presents an integrative review aimed: to map the use of serious games in schoolchildren in promoting self-care in health. The mapping focus was the games analysis of the contribution to learning, knowledge acquisition, skills development and it's own reflection on self-care. The review was conducted from 7 electronic databases. It included the studies published in the last five years, the original ones which focused on the use of digital games with school students, the thematic self-care and health promotion. From the 685 studies reviewed, 5 were included. Despite the large volume of publications making use of serious games, there was a small number relating serious games with learning, self-care and health promotion. However, there are indications of the great potential of serious games in the school environment for health promotion, especially regarding the development of skills for self-care.
\end{abstract}

Keywords: serious games; school health; health promotion; self-care; skills. 


\section{Introdução}

Os jogos digitais com propósito, também conhecidos como "serious games", têm sido amplamente utilizados nos últimos anos como ferramentas educacionais no processo de ensino aprendizagem (Girard, 2013). Estas ferramentas digitais estão presentes de forma massiva na vida dos escolares, os chamados "nativos digitais", e são fonte de motivação e amplo envolvimento (Girard, 2013). Os pesquisadores têm voltado sua atenção para o desenvolvimento de jogos digitais para áreas específicas do currículo escolar como o ensino de línguas (Guillén-Nieto, Aleson-Carbonell 2012), história (Yu, 2014), imunologia (Cheng, 2014) dentre outros.

Nesse sentido, passou-se a explorar a utilização de jogos digitais para temas relacionados à saúde (Baranowski, 2008; Papastergiou, 2009; Wattanasoontorn, 2013), uma vez que existe a dificuldade clara de inserir tais abordagens na matriz curricular escolar (Gomes, 2009). Umas das questões que têm fomentado a preocupação em inserir os cuidados de saúde no âmbito escolar é a elevada prevalência de sobrepeso e obesidade entre as crianças desta faixa etária (Niehues, 2014), predispondo-as precocemente a diversas comorbidades (Niehues, 2014; Carvalho, 2011). Um referencial teórico que tem se destacado nos estudos sobre a promoção da saúde é o da Teoria Social Cognitiva e da Autorregulação para a saúde (Tougas, 2015). Perspectiva essa que reforça o papel ativo do indivíduo na regulação da sua saúde, através das relações que estabelece entre os seus fatores internos (motivação, autorregulação) e o meio (Bandura, 2005).

Frente a este cenário, o presente artigo de revisão teve como objetivo mapear a utilização de jogos digitais entre escolares na promoção do autocuidado em saúde, a fim de oferecer perspectivas quanto à sua contribuição no aprendizado, na aquisição de conhecimento, no desenvolvimento de competências e na própria reflexão sobre o autocuidado em saúde.

\section{Materiais e Métodos}

O presente estudo configura-se como uma revisão bibliográfica sistemática integrativa, composta por seis etapas (Grupo Anima Educação, 2014).

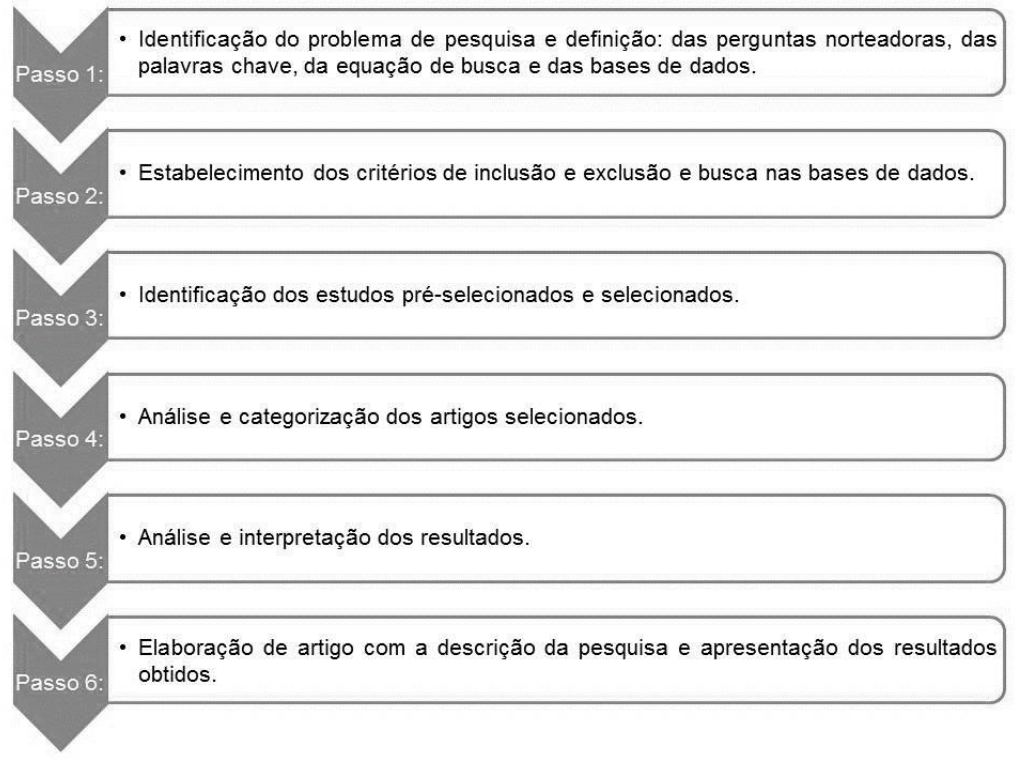

Figura 1 - Etapas da revisão bibliográfica sistemática integrativa. 
Em sua primeira etapa foi identificado o problema e elaborado as perguntas norteadoras da investigação, sendo elas: 1) Os jogos digitais favorecem o aprendizado de escolares no que tange o autocuidado em saúde?; 2) Os jogos digitais favorecem o aumento do conhecimento no autocuidado em saúde?; 3) Os jogos digitais favorecem o desenvolvimento de competências para o autocuidado em saúde?; 4) Os jogos digitais proporcionam uma reflexão sobre o autocuidado em saúde?. Em seguida, definiram-se as palavras chave "serious games", "child" e "health". Após a realização de testes de busca com as palavras chave na plataforma de periódicos Capes foi definida a seguinte equação de busca: (serious games $O R$ digital games) AND child* $A N D$ health. Optou-se por realizar a busca nas bases de dados que retornassem uma maior quantidade de publicações na ferramenta de busca da Capes e que tivessem maior relação com o assunto pesquisado. Desta maneira, foram definidas as bases de dados: Scopus (Elsevier), Science Citation Index Expanded e Social Sciences Citation Index (Web of Science), ERIC (U.S. Dept. of Education), Wiley Online Library, SpringerLink, Directory of Open Access Journal (DOAJ) e Computer and Information Systems Abstracts. Na segunda etapa foram definidos os critérios de inclusão e exclusão conforme descrito na tabela 1 .

Tabela 1 - Passo 2 da revisão integrativa: critérios de inclusão e exclusão.

\begin{tabular}{|c|c|}
\hline Critérios de Inclusão & Critérios de Exclusão \\
\hline Artigo original & Artigo de revisão \\
\hline $\begin{array}{l}\text { Estudo desenvolvido com escolares com idade } \\
\text { entre } 6 \text { e } 17 \text { anos que estejam cursando o ensino } \\
\text { fundamental e/ou ensino médio }\end{array}$ & $\begin{array}{l}\text { Estudos cujo foco não seja a utilização } \\
\text { de jogos digitais como meio de } \\
\text { promover o autocuidado em saúde }\end{array}$ \\
\hline $\begin{array}{l}\text { Estudo cuja intervenção seja através de jogos } \\
\text { digitais }\end{array}$ & $\begin{array}{l}\text { Estudos cujo foco seja a utilização de } \\
\text { jogos digitais para reabilitação de } \\
\text { condições específicas de saúde }\end{array}$ \\
\hline $\begin{array}{l}\text { Estudo cujo desfecho esteja relacionado à } \\
\text { aquisição de conhecimento e/ou } \\
\text { desenvolvimento de competências no } \\
\text { autocuidado em saúde }\end{array}$ & $\begin{array}{l}\text { Estudos que apenas propõem o uso de } \\
\text { jogos digitais para promover o } \\
\text { autocuidado, sem obtenção de } \\
\text { resultados }\end{array}$ \\
\hline Artigos publicados a partir de janeiro de 2011 & $\begin{array}{l}\text { Estudos que façam uso de vídeo } \\
\text { games de movimento (exergames) }\end{array}$ \\
\hline $\begin{array}{l}\text { Artigos em língua espanhola, inglesa ou } \\
\text { portuguesa }\end{array}$ & Artigos duplicados \\
\hline
\end{tabular}

Após leitura e análise de 685 títulos e resumos retornados, foram pré-selecionadas as publicações que possuíam potencial de responder às questões norteadoras e atendiam os critérios de inclusão e exclusão. Restaram 16 publicações para leitura na íntegra. Após criteriosa análise, 3 publicações se mostraram plenamente aptas a responder às perguntas norteadoras da investigação. Todavia, durante a leitura das 16 publicações pré-selecionadas, observou-se que algumas referências possuíam potencial para responder às perguntas, sendo analisadas com cuidado. Desta forma, 2 publicações foram incluídas ao corpo de artigos selecionados, finalizando a terceira etapa da revisão integrativa. 


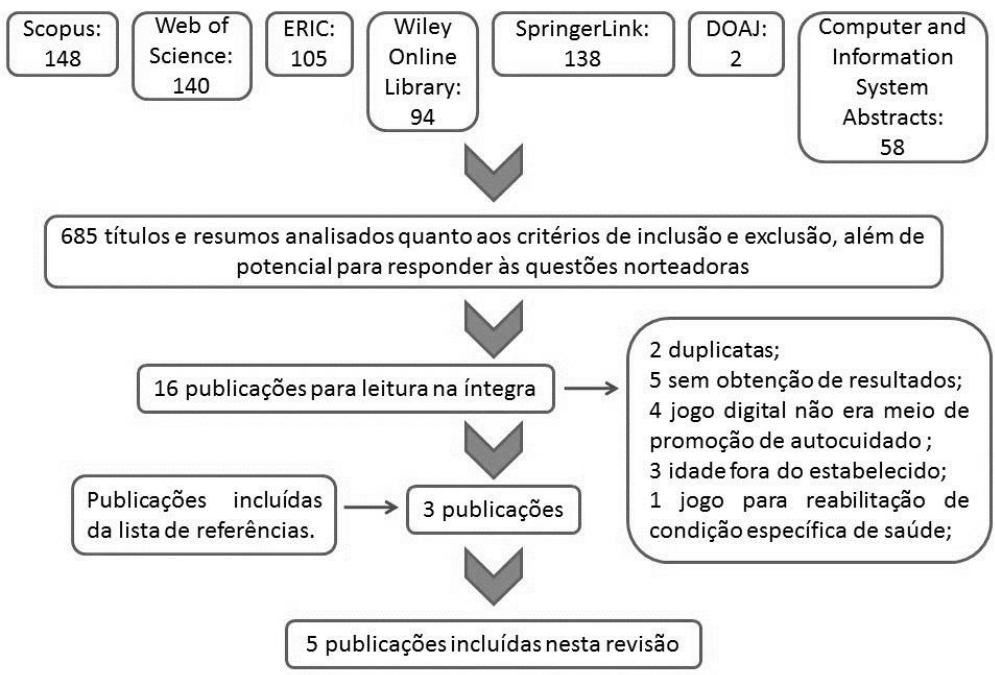

\section{Figura 2 - Fluxograma do passo 3 da revisão integrativa: identificação dos estudos pré-selecionados e selecionados.}

\section{Resultados e Discussão}

Observou-se um grande volume de publicações utilizando-se de jogos digitais, ou "serious games", nas mais variadas abordagens: no ensino de disciplinas específicas tanto na educação básica quanto no ensino superior; na reabilitação em condições específicas e na recuperação da saúde após agravos já instalados; propostas de taxonomia de "serious games". Entretanto, um volume muito pequeno de publicações relacionando jogos digitais com escolares, autocuidado e promoção de saúde. Das 5 publicações que relacionaram estes conteúdos, a maioria $(n=3)$ possui foco na promoção de hábitos alimentares saudáveis, às vezes associado à promoção de atividade física $(\mathrm{n}=2)$. Uma das publicações aborda a prevenção de agravos à saúde e outra a prevenção de doenças transmitidas por alimentos, ou seja, a segurança alimentar.

Em relação às perguntas norteadoras da investigação observou-se que o aspecto mais explorado nas publicações se referiu à capacidade dos jogos digitais favorecerem o desenvolvimento de competências para o autocuidado em saúde, evidenciando o interesse nas implicações práticas que os jogos podem ter na vida dos escolares. $\mathrm{O}$ segundo aspecto mais explorado foi a capacidade dos jogos digitais favorecerem o aumento do conhecimento no autocuidado em saúde. Das 5 publicações, 3 exploraram esse aspecto, que vai ao encontro do desenvolvimento de competências, uma vez que o conhecimento é um dos determinantes para a mudança de comportamento. Já a capacidade dos jogos digitais favorecerem o aprendizado e proporcionarem uma reflexão sobre autocuidado em saúde foram as abordagens menos estudadas. Tal resultado provavelmente se deva à dificuldade de mensurar aprendizagem e reflexão, justamente pelas suas características processuais (tabela 3). 
Tabela 3 - Passo 4 da revisão integrativa: análise e categorização dos artigos selecionados.

\begin{tabular}{|c|c|c|c|c|}
\hline \multirow[b]{2}{*}{$\begin{array}{c}\text { Publicações } \\
\text { Analisadas }\end{array}$} & \multicolumn{3}{|c|}{ Os jogos digitais favorecem: } & \multirow[b]{2}{*}{$\begin{array}{c}\text { Os jogos digitais } \\
\text { proporcionam } \\
\text { uma reflexão } \\
\text { sobre o } \\
\text { autocuidado em } \\
\text { saúde? }\end{array}$} \\
\hline & $\begin{array}{c}\text { O aprendizado } \\
\text { sobre } \\
\text { autocuidado } \\
\text { em saúde? }\end{array}$ & $\begin{array}{c}\text { O aumento do } \\
\text { conhecimento } \\
\text { no } \\
\text { autocuidado } \\
\text { em saúde? }\end{array}$ & $\begin{array}{c}\text { O } \\
\text { desenvolvimento } \\
\text { de competências } \\
\text { para o } \\
\text { autocuidado em } \\
\text { saúde? } \\
\end{array}$ & \\
\hline Yang (2015) & Sim. & $\begin{array}{c}\text { Abordagem não } \\
\text { explorada. }\end{array}$ & Sim. & $\begin{array}{c}\text { Abordagem } \\
\text { não explorada. }\end{array}$ \\
\hline Sung (2015) & $\begin{array}{c}\text { Abordagem não } \\
\text { explorada. }\end{array}$ & Sim. & Sim. & $\begin{array}{c}\text { Abordagem } \\
\text { não explorada. }\end{array}$ \\
\hline $\begin{array}{l}\text { Quick } \\
\text { (2013) }\end{array}$ & $\begin{array}{c}\text { Abordagem não } \\
\text { explorada. }\end{array}$ & Sim. & Sim. & Sim. \\
\hline $\begin{array}{l}\text { Majumdar } \\
\text { (2013) }\end{array}$ & $\begin{array}{c}\text { Abordagem não } \\
\text { explorada. }\end{array}$ & $\begin{array}{c}\text { Abordagem não } \\
\text { explorada. }\end{array}$ & Sim. & $\begin{array}{c}\text { Abordagem } \\
\text { não explorada. }\end{array}$ \\
\hline $\begin{array}{l}\text { Schneider } \\
\text { (2012) }\end{array}$ & $\begin{array}{c}\text { Abordagem não } \\
\text { explorada. }\end{array}$ & Sim. & Sim. & $\begin{array}{c}\text { Abordagem } \\
\text { não explorada. }\end{array}$ \\
\hline
\end{tabular}

Estudos com jogos digitais são escassos, ainda mais quando se considera a perspectiva da promoção da saúde entre o público infanto-juvenil saudável. Em revisão realizada por Baranowski (2008), foram encontradas 9 publicações que faziam uso de jogos digitais com crianças para a promoção da saúde através de mudanças na alimentação e da prática de atividade física. Já na revisão realizada por Papastergiou (2009) também foram encontradas 9 publicações utilizando-se de jogos digitais para a promoção da saúde infanto-juvenil através de mudanças na alimentação, da prática de atividade física, no autocuidado com a pele a fim de evitar o desenvolvimento de cânceres e no autocuidado quando da queda na prática de atividades esportivas.

Para a análise dos 5 artigos que compõem o corpo documental desta revisão, foi considerado aprendizagem o processo de aquisição de conhecimento com interação dos recursos cognitivos, motivacionais e emocionais (Rosário, 2012). Nenhuma publicação se propôs a avaliar a aprendizagem objetivamente. Entretanto, Yang (2015) avaliou a ingestão alimentar dos escolares pré-intervenção, pós-intervenção e durante dois momentos da intervenção, envolvendo diferentes etapas do jogo, possibilitando assim, a observação da melhora progressiva da ingestão alimentar em termos nutricionais. Tal processo de avaliação proporciona subsídios para inferir-se que, se houve um processo de alteração da ingestão alimentar, supõe-se ter havido um processo de aprendizagem que proporcionou as condições para a alteração deste comportamento.

Já o conhecimento foi considerado como uma das dimensões da aprendizagem cuja tipificação contempla conhecimento declarativo, procedimental e condicional (Rosário, 2007; Rosário 2012). Sung (2015) avaliou conhecimento através de pré-teste e pós-teste. Todavia, utilizou-se de uma abordagem diferenciada: no pré-teste avaliou concepções básicas de cuidados com a saúde e o pós-teste foi dividido em concepções básicas acrescido de questões para medir conhecimento avançado para escolha de diferentes estratégias de saúde, demonstrando que o jogo digital avaliado na intervenção foi capaz de proporcionar uma evolução do conhecimento. Já Quick (2013) e Schneider (2012) utilizaram-se de metodologia semelhante: questionário com questões de múltipla escolha aplicado no pré-teste e pós-teste, demonstrando aumento 
do número de acertos após a aplicação dos jogos, evidenciando aumento do conhecimento declarativo.

Segundo a Teoria Social Cognitiva, referencial teórico utilizado para conceber este artigo, competências são capacidades que uma pessoa possui em determinadas áreas, sejam elas, por exemplo, instrumentais, interpessoais ou sistêmicas (Rosário, 2012). Todos os trabalhos analisados permitem verificar que os jogos digitais favorecem o desenvolvimento de competências. Entretanto, nos estudos revisados, as competências analisadas foram bastante diversas, bem como as formas de analisá-las. Yang (2015) e Majumdar (2013) avaliaram a competência de realizar uma alimentação saudável. Majumdar (2013) acrescentou ainda as competências de ingerir água e praticar atividade física em níveis considerados saudáveis.

Ambos estudos citados avaliaram a competência de realizar uma alimentação saudável através do consumo alimentar. Yang (2015) utilizou-se de abordagem objetiva, através do registro diário dos alimentos consumidos e sua análise em relação à recomendação das porções dos grupos alimentares e quantidades de macro e micronutrientes adequadas. Verificou, assim, que o jogo afetou positivamente o consumo alimentar, mostrando-se eficaz no desenvolvimento da competência avaliada. Já Majumdar (2013) utilizou-se de um questionário de frequência alimentar semi-quantitativo para alimentos específicos como "lanches de pacote" e "bebidas adoçadas". O jogo proposto mostrou-se eficaz no desenvolvimento da competência de alimentação saudável em virtude da diminuição da quantidade e frequência relatadas do consumo destes alimentos, não sendo eficaz no desenvolvimento das outras competências avaliadas: consumo de água e prática de atividade física. Sung (2015) explorou em seu jogo a competência de resolução de problemas, utilizando-se como referência a teoria da aprendizagem contextual e afirmando que através de um jogo que explorasse contextos autênticos de problemas de saúde era possível aprender conteúdos de saúde. Sob esta perspectiva, o jogo demonstrou favorecer o desenvolvimento da competência de resolução de problemas quando comparado à utilização de e-book como única ferramenta de aprendizagem.

Schneider (2012) e Quick (2013) não avaliaram uma competência diretamente, porém, avaliaram auto-eficácia para comportamentos específicos, que pode ser considerada um indício de desenvolvimento de competência, pois constitui a maior base para a ação (Bandura, 1998). Schneider (2012) avaliou, através de questionários, a auto-eficácia para alimentação saudável bem como atitudes relacionadas à alimentação saudável, encontrando efeito positivo em ambos os quesitos através do jogo. Já Quick (2013) avaliou, também através de questionários, a auto-eficácia para a manipulação segura de alimentos, a intenção e a frequência de comportamentos habituais relacionados à manipulação segura, obtendo sucesso em todos os componentes através do jogo proposto.

Por fim, para responder à última pergunta: se os jogos digitais proporcionam uma reflexão sobre o autocuidado em saúde, utilizou-se como referência os pensamentos de Schön (2007) que explora a reflexão como o ato de pensar sobre o que está sendo realizado enquanto se realiza. Acredita-se que devido à dificuldade de mensurar a reflexão, nenhum estudo teve como objetivo avaliar o desenvolvimento de processo reflexivo. Contudo, dois trabalhos chamaram a atenção: Sung (2015) através da avaliação de conhecimento básico e conhecimento avançado, permitindo ao leitor se questionar se houve um processo de reflexão durante a intervenção com o jogo que auxiliou no processo de evolução de conhecimento. Todavia, o estudo não explora esta abordagem nem fornece mais subsídios. O estudo de Quick (2013) mensurou a 
percepção de risco/susceptibilidade às doenças transmitidas por alimentos, que aumentou após a intervenção com o jogo. Para Bandura (1998), esta susceptibilidade representa um resultado físico negativo esperado no modelo de crenças de saúde e que permite a inferência de que houve um questionamento interno sobre o que estava sendo realizado no jogo enquanto ele era jogado e que forneceu subsídios suficientes para os escolares se questionarem quanto às suas práticas reais e perceberem de uma forma mais madura a sua susceptibilidade às doenças transmitidas por alimentos.

Algumas considerações podem ser feitas quanto às metodologias dos estudos e os aspectos explorados. Observou-se que apenas um estudo (Quick, 2013) aplicou uma medida de follow up de 1 semana, período muito curto. Principalmente levando-se em consideração que todos os estudos avaliaram o desenvolvimento de competências, sendo essencial, portanto, o acompanhamento por um período mais longo de tempo para verificar o real desenvolvimento da competência. Além disso, observou-se também que apenas um estudo (Yang, 2015) avaliou processo, isto é, realizou medidas repetidas durante a intervenção. A análise de medidas repetidas durante a intervenção constitui-se ferramenta de grande valor na área estudada, uma vez que permite avaliar as modificações que vão se desenvolvendo tanto em relação aos processos de aprendizagem, quanto de reflexão e do próprio desenvolvimento de competências. Outra questão que chamou bastante atenção foi a diversificação na duração das intervenções, variando de 1 a 8 semanas. O número de vezes nas quais os escolares foram expostos aos jogos também variou bastante. Sendo mais constante apenas o período de exposição, que foi de 30 a 50min. Em relação ao desenvolvimento de competências, observou-se que os jogos que se propuseram a desenvolver mais de uma competência acabaram se mostrando efetivos em apenas uma delas, na competência melhor explorada no jogo. Por conseguinte, parece mais interessante o jogo possuir como foco o desenvolvimento de apenas uma competência e explorá-la ao máximo do que tentar desenvolver diversas competências em uma concepção de jogo muito complexa, mesmo que as competências sejam bastante relacionadas: como alimentação saudável, ingestão recomendada de água e atividade física.

\section{Conclusões}

Assim sendo, considerando o resultado do presente estudo, apresenta-se mais uma evidência na direção do potencial dos jogos digitais favorecerem o desenvolvimento de competências para o autocuidado em saúde no ambiente escolar. Contudo, há de ser considerado o universo muito pequeno de publicações sobre o assunto, como sendo uma limitação deste estudo. Como contribuição deste artigo identifica-se um nicho de pesquisa em relação à utilização de jogos digitais entre escolares para o autocuidado em saúde na perspectiva da promoção da saúde utilizando-se de intervenções com duração mais prolongada, período de follow up mais extenso e realização de medidas repetidas durante a intervenção para explorar os processos de aprendizagem mobilizados pelos jogos digitais.

\section{Referências}

Bandura A. Health promotion from the perspective of social cognitive theory. Psychology and Health 1998; 13(4):623-49. Disponível em: http://www.tandfonline.com/doi/abs/10.1080/08870449808407422 Acesso em: 7 jan. 2016 
Bandura A. The Primacy of Self- Regulation in Health Promotion. Applied Psychology 2005 Abr; 54(2):245-54. Disponível em: http://onlinelibrary.wiley.com/doi/10.1111/j.14640597.2005.00208.x/abstract?userIsAuthenticated $=$ false \&deniedAccessCustomisedMess age Acesso em: 7 jan. 2016

Baranowski T, Buday R, Thompson DI, Baranwski J. Playing for Real: Video Games and Stories for Health-Related Behavior Change. American Journal of Preventive Medicine 2008 Jan; 34(1):74-82 e10. Disponível em: http://www.sciencedirect.com/science/article/pii/S0749379707006472 Acesso: 7 jan. 2016

Carvalho SG. Obesidade infantil, a epidemia do séc.XXI a revisão da literatura sobre novas estratégias de prevenção [dissertação]. Instituto de Ciências Biomédicas Abel Salazar (ICBAS): Universidade do Porto; 2011. Disponível em: https://repositorioaberto.up.pt/handle/10216/21125 Acesso em: 7 jan. 2016

Cheng MT, Su T, Huang WY, Chen JH. An educational game for learning human immunology: What do students learn and how do they perceive? British Journal of Educational Technology 2014 Set; 45(5):820-33. Disponível em: http://onlinelibrary.wiley.com/doi/10.1111/bjet.12098/abstract?userIsAuthenticated=fal se\&deniedAccessCustomisedMessage Acesso em: 7 jan. 2016

Girard C, Ecalle J, Magnan A. Serious games as new educational tools: how effective are they? A meta-analysis of recent studies. Journal of Computer Assisted Learning 2013 Jun; 29(3):207-19. Disponível em: http://onlinelibrary.wiley.com/doi/10.1111/j.13652729.2012.00489.x/abstract;jsessionid=A68397348934A09ED07B9D546CEFF8BA.f0 1t03? userIsAuthenticated=false\&deniedAccessCustomisedMessage Acesso em: 7 jan. 2016

Gomes JP. As Escolas Promotoras de Saúde: uma via para promover a saúde e a educação para a saúde da comunidade escolar. Educação 2009; 32(1):84-91. Disponível

em: http://revistaseletronicas.pucrs.br/ojs/index.php/faced/article/viewArticle/5229 Acesso em: 7 jan. 2016

Grupo Anima Educação. Manual Revisão Bibliográfica Sistemática Integrativa: a pesquisa baseada em evidências. Belo Horizonte: Grupo Anima Educação; 2014. 58p. Guillén-Nieto V, Aleson-Carbonell M. Serious games and learning effectiveness: The case of It's a Deal! Computers \& Education 2012 Jan; 58(1):435-48. Disponível em: http://www.sciencedirect.com/science/article/pii/S0360131511001734 Acesso: 7 jan. 2016

Majumdar D, Koch PA, Lee H, Contento IR, Islas-Ramos AL, Fu D. " Creature- 101": A Serious Game to Promote Energy Balance- Related Behaviors Among Middle School Adolescents. Games For Health Journal 2013 Out 21; 2(5):280-90. Disponível em: http://online.liebertpub.com/doi/abs/10.1089/g4h.2013.0045 Acesso em: 7 jan. 2016

Niehues JR, Gonzales AI, Lemos RR, Bezerra PP, Haas P. Prevalence of overweight and obesity in children and adolescents from the age range of 2 to 19 years old in Brazil. International Journal of Pediatrics 2014 Jun; 2014. Disponível em: http://www.hindawi.com/journals/ijpedi/2014/583207/cta/ Acesso em: 7 jan. 2016 Papastergiou M. Exploring the potential of computer and video games for health and physical education: A literature review. Computers \& Education 2009 Nov; 53(3):603-22. $\quad$ http://www.sciencedirect.com/science/article/pii/S0360131509000840 Acesso em: 7 jan. 2016 
Quick V, Corda KW, Chamberlin B, Schaffner DW, Byrd-Bredbenner C. Ninja Kitchen to the rescue: Evaluation of a food safety education game for middle school youth. British Food Journal 2013; 115(5):686-99. Disponível em: http://www.emeraldinsight.com/doi/abs/10.1108/00070701311331481 Acesso: 7 jan. 2016

Rosário P, Polydoro S. Capitanear o Aprender: Promoção da autorregulação da aprendizagem no contexto escolar. 1 ed. São Paulo: Casa do Psicólogo; 2012.

Rosário PSL, Nuñez JC, González-Pienda J. Auto-regulação em crianças sub-10: Projecto Sarilhos do Amarelo. Porto: Porto Editora; 2007.

Schneider KL, Ferrara J, Lance B, Karetas A, Druker S, Panza E, et al. Acceptability of an Online Health Videogame to Improve Diet and Physical Activity in Elementary School Students: " Fitter Critters". Games For Health Journal 2012 Ago 10; 1(4):26268. Disponível em: http://online.liebertpub.com/doi/abs/10.1089/g4h.2012.0009 Acesso em: 7 jan. 2016

Schön DA. Educando o professional reflexive [recurso eletrônico]: um novo design para o ensino e a aprendizagem. Porto Alegre: Artmed; 2007.

Sung HY, Hwang GJ, Yen YF. Development of a contextual decision-making game for improving students' learning performance in a health education course. Computers \& Education 2015 Mar; 82:179-90. Disponível em: http://www.scopus.com/inward/record.url?eid=2-s2.084916877789\&partnerID=40\&md5=d1c5ff30b1867f9038f6960e3a7c83a3 Acesso em: 7 jan. 2016

Tougas ME, Hayden JA, McGrath PJ, Huguet A, Rozario S. A Systematic Review Exploring the Social Cognitive Theory of Self-Regulation as a Framework for Chronic Health Condition Interventions. PLoS One 2015 Ago; 10(8): e0134977. Disponível em: http://journals.plos.org/plosone/article?id=10.1371/journal.pone.0134977 Acesso em: 7 jan. 2016

Wattanasoontorn V, Boada I, García R, Sbert M. Serious games for health. Entertainment Computing 2013 Dez; 4(4):231-47. Disponível em: http://www.scopus.com/inward/record.url?eid=2-s2.0$\underline{84887574617 \& \text { partnerID }=40 \& \mathrm{md} 5=\mathrm{e} 6 \mathrm{~b} 5 \mathrm{e} 936 \mathrm{edd} 89376563501 \mathrm{e} 5 \mathrm{f} 948852 \mathrm{~d}}$ Acesso em: 7 jan. 2016

Yang YTC, Wang CJ, Tsai MF, Wang JS. Technology-enhanced game-based team learning for improving intake of food groups and nutritional elements. Computers \& Education 2015 Out; 88:143-59. Disponível em: http://www.scopus.com/inward/record.url?eid=2-s2.084930661883\&partnerID=40\&md5=161cce0946f5e953fe7223d1272077dd Acesso em: 7 jan. 2016

Yu Z, Yu WH, Fan X, Wang X. An exploration of computer game-based instruction in the "world history" class in secondary education: A comparative study in China. PLoS ONE 2014 Mai; 9(5). Disponível em: http://www.scopus.com/inward/record.url?eid=2-s2.084901218204\&partnerID=40\&md5=3b8d815bb9fdff5adc59cb1671e8fc46 Acesso em: 7 jan. 2016 